\title{
Absorption spectra of irradiated XRCT radiochromic film
}

\author{
Martin J Butson ${ }^{1,2}$, Tsang Cheung ${ }^{1}$ and Peter K N Yu ${ }^{1}$ \\ ${ }^{1}$ Department of Physics and Materials Science, City University of Hong Kong, Kowloon Tong, \\ Hong Kong \\ ${ }^{2}$ Department of Medical Physics, Illawarra Cancer Care Centre, Crown St, PO Box 1798 , \\ Wollongong, NSW 2500, Australia \\ E-mail: butsonm@iahs.nsw.gov.au
}

Received 9 February 2006

Published 31 May 2006

Online at stacks.iop.org/PMB/51/3099

\begin{abstract}
Gafchromic XRCT radiochromic film is a self-developing high sensitivity radiochromic film product which can be used for assessment of delivered radiation doses which could match applications such as computed tomography (CT) dosimetry. The film automatically changes colour upon irradiation changing from a yellow to green/brown colour. The absorption spectra of Gafchromic XRCT radiochromic film as measured with reflectance spectrophotometry have been investigated to analyse the dosimetry characteristics of the film. Results show two main absorption peaks produced from irradiation located at $636 \mathrm{~nm}$ and $585 \mathrm{~nm}$. This is similar to EBT Gafchromic film. A high level of sensitivity is found for this film with a $1 \mathrm{cGy}$ applied dose producing an approximate net optical density change of 0.3 at $636 \mathrm{~nm}$. This high sensitivity combined with its relatively energy independent nature around the $100 \mathrm{kVp}$ to $150 \mathrm{kVp}$-ray energy range provides a unique enhancement in dosimetric measurement capabilities over currently available dosimetry films for CT applications.
\end{abstract}

\section{Introduction}

Manufacturers have been continually improving the sensitivity of radiochromic film products to X-ray radiation (Cheung et al 2000, Nariyama et al 2002, Butson et al 2002) in pursuit of providing accurate dosimetry tools for low dose applications such as computed tomography dose assessment. Radiochromic film has been extensively used over the past few years for industrial and therapeutic applications (Butson et al 2003, Vuong et al 2003, Meigooni et al 1996, Devic et al 2004) where higher dose levels are normally delivered. In the last few years, International Specialty Products Inc (ISP Corp) has developed radiochromic films suited for analysis in the lower $\mathrm{X}$-ray energy range relating to areas such as diagnostic $\mathrm{X}$-ray machines, CT 
and superficial/orthovoltage therapy (ISP Corp 2006). One such film is Gafchromic XRCT. This has been made possible due to higher dose sensitivities and a film emulsion, which allows reflective assessment of absorbed dose. Two major constraints on accurate dosimetry for low energy x-rays such as those produced by CT machines (Bahar Gogani et al 2005) are the energy response of the dosimeter and the sensitivity. Radiographic films, which require processing through a chemical development and fixing process, are notoriously energy dependent and require complex energy correction calculations (Kron et al 1998). Most Gafchromic film products have relatively low energy dependence but are also relatively insensitive to radiation and do not match the requirements for low dose assessment. XRCT film exhibits a relatively energy independent (Butson et al 2006) response to $\mathrm{x}$ radiation in the $100 \mathrm{kVp}$ to $150 \mathrm{kVp}$ energy range and also is significantly more sensitive. This paper investigates the absorption spectrum of Gafchromic XRCT film when irradiated with kilovoltage x-ray radiation.

\section{Materials and methods}

Gafchromic XRCT radiochromic film (Lot No. 3514600728XRCT) was used for the reflectance absorption spectra study. For dose delivery, the films were positioned in a solid water (Constantinou et al 1982) phantom of dimensions $30 \mathrm{~cm} \times 30 \mathrm{~cm} \times 30 \mathrm{~cm}$. The phantom was placed on a Gulmay D3300 superficial/orthovoltage x-ray machine and doses ranging from $0 \mathrm{cGy}$ to $20 \mathrm{cGy}$ according to the IPEMB protocol (IPEMB 1996) were given using a $125 \mathrm{kVp}(54 \mathrm{keV}$ effective energy) $\mathrm{x}$-ray beam. The therapeutic $\mathrm{x}$ ray machine was chosen to deliver radiation doses due to its accurate dose calibration and ability to deliver at kilovoltage energies. The films were irradiated placed perpendicular to the central axis of the beam. Standard precautions in handling radiochromic films such as not touching active film surfaces and keeping film temperature controlled as outlined in TG-55 were used (Niroomand-Rad et al 1998). Absorption spectra results were measured using an Avantes AvaSpec-2048 reflectance spectrophotometer. The AvaSpec-2048 device is a fibre optic Spectrometer with a 300 lines $\mathrm{mm}^{-1}$ grating. The bandwidth of operation is from $327 \mathrm{~nm}$ to $1100 \mathrm{~nm}$ and the light source has a FWHM resolution of $2.4 \mathrm{~nm}$. Measurements were made in absorbance mode, which provides directed measurement of reflected 'optical density' (Cheung et al 2005) in absorbance units. From these results a set of absorption spectra measurements was obtained over the wavelength region of $530 \mathrm{~nm}$ to $660 \mathrm{~nm}$, which includes the two main absorption, peaks of XRCT radiochromic film. These absorption spectra have also been integrated over various band passes and wavelengths to provide analysis of dose response over various standard densitometer configurations, namely at the $636 \mathrm{~nm}$ absorption peak, the $632.8 \mathrm{~nm} \mathrm{HeNe}$ scanning wavelength, the $530 \mathrm{~nm}-$ $660 \mathrm{~nm}$ white light equivalent and the $600 \mathrm{~nm}-660 \mathrm{~nm}$ red component of an RGB image.

\section{Results and discussion}

Figure 1 shows the optical density absorption spectra for Gafchromic XRCT film in the wavelength region of 530 to $660 \mathrm{~nm}$. Results are given for films ranging in applied doses of $0 \mathrm{cGy}$ to $20 \mathrm{cGy}$ produced by a $125 \mathrm{kVp}$ x-ray beam. As can be seen the film produces two marked absorption peaks upon irradiation, which are located at $636 \mathrm{~nm}$ and $585 \mathrm{~nm}$. These two absorption peaks are similar to another Gafchromic film product, EBT (Butson et al 2005). Two major differences are seen in the absorption spectra for this product compared to EBT, these being: (1) the large increase in absorption below wavelengths around $550 \mathrm{~nm}$ and (2) the large increase in sensitivity to dose, i.e. approximately 20 times as sensitive (Butson 


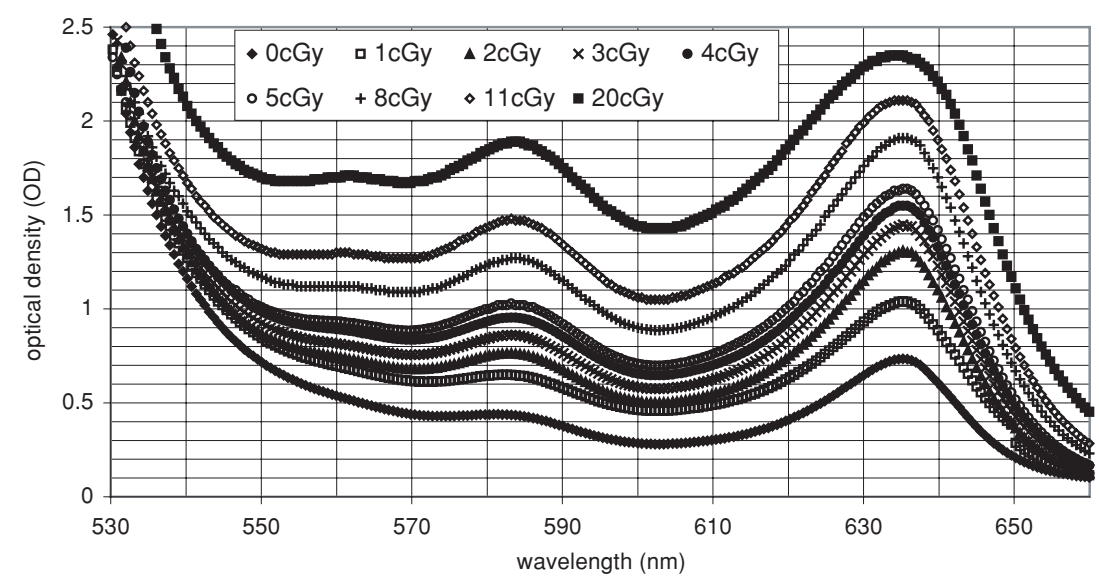

Figure 1. The absorption spectra for XRCT radiochromic film exposed to $125 \mathrm{kVp}$-rays (54 keV effective).

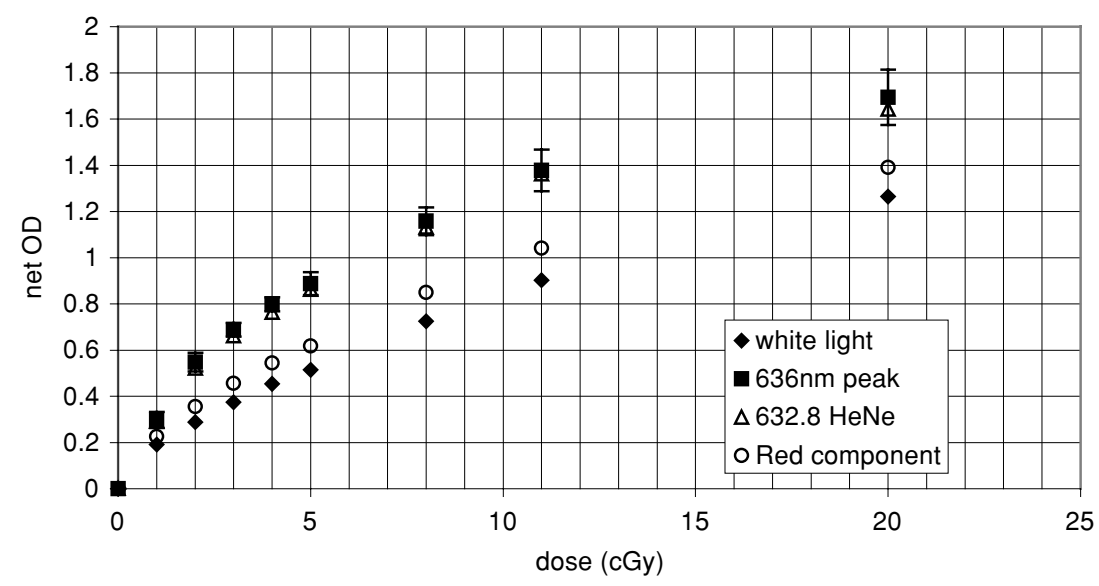

Figure 2. Dose response curves for XRCT radiochromic film when analysed at various wavelengths or band passes.

et al 2005). The increased absorption below wavelengths of $550 \mathrm{~nm}$ is due to the coloured dye of the overlaying material used in the construction of XRCT film. The manufacturers have added a yellow dye to the construction which gives the film its initial yellow colour. This aids to increase the visual sensitivity of the colour change seen upon irradiation. The increase in dose sensitivity is most likely due to a change in chemical composition of the film emulsion to enhance the dose response at lower kilo voltage $\mathrm{x}$-ray energies and to produce a relatively energy independent response at CT diagnostic x-ray beam energies. This is normally performed with the use of higher atomic number atoms or molecules which enhances the photoelectric attenuation of the emulsion.

Figure 2 shows the dose response of the film at specific wavelengths and band passes of light. Results are quoted as net optical density change versus applied dose. Net optical density is the change in visible optical density of the film from its unirradiated to irradiated state. As can be seen, due to the variation in absorption at specific wavelengths, the dose response curves are different when analysed at different wavelength regions. At all wavelengths there 
is a nonlinear response with dose which reduces the change in optical density with dose as the film becomes darker which is most likely due to the active emulsion's saturation level. At the absorption peak wavelength of $636 \mathrm{~nm}$, an approximate net optical density change of 0.3 is seen for $1 \mathrm{cGy}$ applied dose which reduces to an average 0.137 net OD per $1 \mathrm{cGy}$ after $10 \mathrm{cGy}$ applied dose. Error bars on the $636 \mathrm{~nm}$ peak data show the variation in response for 10 film pieces to $95 \%$ confidence. Similar uncertainties are measured for the different wavelengths of analysis but are not included in the figure to improve clarity of presentation. Results also show that depending on the wavelength of analysis various sensitivity or dose response functions can be obtained, with a white light average producing a net OD change of 0.9 at $10 \mathrm{cGy}$ applied dose versus a 1.37 change in OD for $636 \mathrm{~nm}$ wavelength analysis. These results show a much higher sensitivity to radiation as compared to traditional radiochromic film products such as Gafchromic HS, EBT and XR type R, which require approximately $1000 \mathrm{cGy}$ (Butson et al 2002), $200 \mathrm{cGy}$ (Butson et al 2005) and $170 \mathrm{cGy}$ (Cheung et al 2005) to achieve a net change in OD of 1 at their peak absorption wavelengths, respectively. As such, XRCT Gafchromic film produces a much higher sensitivity than other radiochromic film products. This quality, together with its relatively energy independent dose assessment feature (Butson et al 2006), makes it an appealing tool for assessment of low radiation doses at kilovoltage X-ray energies which would prove beneficial for dose measurement in applications such as CT dosimetry.

\section{Conclusion}

Gafchromic XRCT radiochromic film produces a visible colour change upon irradiation and exhibits a high dose sensitivity when compared to other radiochromic film types. Up to 0.3 net OD change per 1 cGy sensitivity is seen with this film at the peak absorption wavelength. This is approximately 30 times more sensitive than EBT Gafchromic film, which is used extensively in therapeutic applications. The absorption spectra show absorption peaks located at $636 \mathrm{~nm}$ and $585 \mathrm{~nm}$. Due to its high sensitivity and combined with a low energy dependence (Butson et al 2006), XRCT film will provide a new tool for dose assessment in low dose applications such as CT X-ray dosimetry.

\section{Acknowledgment}

This work has been fully supported by a grant from the Research Grants Council of HKSAR, China (project no. CityU 100404).

\section{References}

Bahar Gogani J, Hagglund P and Wickman G 2005 Assessment of correlated dose and sensitivity profiles on a multi-slice CT scanner Radiat. Prot. Dosim. 114 332-6

Butson M, Cheung T and Yu P K 2005 Absorption spectra variations of EBT radiochromic film from radiation exposure Phys. Med. Biol. 50 N135-40

Butson M, Cheung T and Yu P K 2006 Measurement of energy dependence for XRCT radiochromic film Med. Phys. submitted

Butson M J, Yu P K, Cheung T and Metcalfe P 2002 High sensitivity radiochromic film dose comparisons Phys. Med. Biol. 47 N291-5

Butson M J, Yu K N, Cheung T and Metcalfe P E 2003 Radiochromic film for Medical Radiation Dosimetry Mater. Sci. Eng. R 41 61-120

Cheung T, Butson M J and Yu P K 2005 Reflection spectrometry analysis of irradiated GAFCHROMIC XR type R radiochromic films Appl. Radiat. Isot. 63 127-9

Cheung Y C, Yu K N, Ho R T and Yu C P 2000 Stereotactic dose planning system used in Leksell Gamma Knife model-B: EGS4 Monte Carlo versus GafChromic films MD-55 Appl. Radiat. Isot. 53 427-30 
Constantinou C, Attix F and Paliwal B 1982 A solid water phantom material for radiotherapy X-ray and gamma ray beam ray calculations Med. Phys. 9 436-41

Devic S, Seuntjens J, Hegyi G, Podgorsak E B, Soares C G, Kirov A S, Ali I, Williamson J F and Elizondo A 2004 Dosimetric properties of improved GafChromic films for seven different digitizers Med. Phys. 31 2392-401

IPEMB 1996 The IPEMB code of practice for the determination of absorbed dose for x-rays below $300 \mathrm{kV}$ generating potential ( $0.035 \mathrm{~mm} \mathrm{Al}-4 \mathrm{~mm} \mathrm{Cu} \mathrm{HVL} ; 10-300 \mathrm{kV}$ generating potential). Institution of Physics and Engineering in Medicine and Biology Phys. Med. Biol. 41 2605-25

ISP Corp 2006 http://www.ispcorp.com

Kron T, Duggan L, Smith T, Rosenfeld A, Butson M, Kaplan G, Howlett S and Hyodo K 1998 Dose response of various radiation detectors to synchrotron radiation. Phys. Med. Biol. 43 3235-59

Meigooni A S, Sanders M F, Ibbott G S and Szeglin S R 1996 Dosimetric characteristics of an improved radiochromic film Med. Phys. 23 1883-8

Nariyama N, Namito Y, Ban S and Hirayama H 2002 Response of GafChromic MD-55 radiochromic film to synchrotron radiation Radiat. Prot. Dosim. $100349-52$

Niroomand-Rad A, Blackwell C, Coursey B, Gall K, Galvin J, McLaughlin W, Meigooni A, Nath R, Rodgers J and Soares C 1998 Radiochromic film dosimetry: Recommendation of AAPM radiation therapy task group 55 Med. Phys. 25 2093-115

Vuong T, Devic S, Belliveau P, Muanza T and Hegyi G 2003 Contribution of conformal therapy in the treatment of anal canal carcinoma with combined chemotherapy and radiotherapy: results of a Phase II Int. J. Radiat. Oncol. Biol. Phys. 56 823-31 